Itinéraires Itinéraires

Littérature, textes, cultures

\title{
Chapitrer ses Mémoires : l'exemple de Simone de Beauvoir
}

«Il est arbitraire de découper sa vie en tranches »

Forms and Issues of Chapters in the Memoirs Genre: The Example of Simone de

Beauvoir

Hélène Baty-Delalande

\section{OpenEdition}

Journals

Édition électronique

URL : http://journals.openedition.org/itineraires/7376

DOI : $10.4000 /$ itineraires.7376

ISSN : 2427-920X

Éditeur

Pléiade

Référence électronique

Hélène Baty-Delalande, « Chapitrer ses Mémoires : l'exemple de Simone de Beauvoir », Itinéraires [En ligne], 2020-1 | 2020, mis en ligne le 02 octobre 2020, consulté le 13 novembre 2020. URL : http:// journals.openedition.org/itineraires/7376 ; DOI : https://doi.org/10.4000/itineraires.7376

Ce document a été généré automatiquement le 13 novembre 2020.

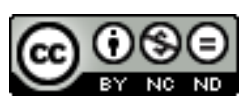

Itinéraires est mis à disposition selon les termes de la licence Creative Commons Attribution - Pas d'Utilisation Commerciale - Pas de Modification 4.0 International. 


\title{
Chapitrer ses Mémoires : l'exemple de Simone de Beauvoir
}

\author{
«Il est arbitraire de découper sa vie en tranches » \\ Forms and Issues of Chapters in the Memoirs Genre: The Example of Simone de \\ Beauvoir
}

Hélène Baty-Delalande

1 La réflexion prendra ici la forme d'une étude de cas, monographique mais cependant moins monolithique qu'on aurait pu s'y attendre : l'importance du dispositif capitulaire dans une série de récits factuels, autobiographiques et mémoriels, publiés par Simone de Beauvoir entre 1958 et 1981, comprenant donc les Mémoires d'une jeune fille rangée, $L a$ Force de l'âge et La Force des choses, Tout compte fait, Une mort très douce et La Cérémonie des adieux. Si les récits de soi ont souvent donné lieu à des analyses fondamentales touchant aux enjeux rhétoriques, éthiques et esthétiques de leur composition, la question du chapitre n'a pas été explicitement abordée pour ce type de corpus. Or, l'un des caractères essentiels du récit de vie réside dans sa double polarisation, d'un point de vue cognitif et narratif : il articule en effet rétrospection mémorielle et prospection narrative. Sa dynamique est ainsi aimantée à la fois par la question de l'origine et par celle de l'aboutissement, ce qui rend le balisage du récit particulièrement crucial. Si l'on admet provisoirement que l'autobiographie comme les Mémoires, genres factuels, reconfigurent l'effectif, l'advenu, donnant forme au divers, inventant un sens à partir de la confusion des jours passés sans pour autant la recouvrir, entretenant la curiosité du lecteur plutôt que ménageant le suspens, on peut supposer que le chapitre n'y a pas tout à fait la même fonction que dans un récit fictif. En tant que dispositif segmentant le récit, il reste cependant un lieu privilégié d'articulation, sinon de négociation, entre la totalisation partielle d'une expérience, et sa mise en perspective à l'échelle du récit tout entier. 


\section{Périodes tranchées : la (ré)-capitulation des Mémoires}

2 "Il est arbitraire de découper sa vie en tranches» (Beauvoir [1960] 2018, I : 688), écrit Beauvoir à la fin de la première partie de La Force de l'âge, faisant le bilan à la fois des dix années qu'elle vient d'évoquer, de 1929 qui marque le passage à l'âge adulte de la toute jeune agrégée de philosophie, compagne de Sartre, à 1939, et le basculement dans la guerre. Et pourtant, comment se raconter autrement qu'en composant, en disposant, en reconfigurant? Et pourtant, ces tranches, loin d'être arbitraires, ne sont-elles pas le lieu même d'une rationalisation du contingent, à travers des ressorts traditionnellement attachés à la fiction? Beauvoir y revient, dans La Force des choses, au milieu de son récit, entre les deux grandes parties qui le composent : «Pourquoi cette pause, soudain? Je sais fort bien qu'une existence ne se décompose pas en périodes tranchées et 1952 n'a pas marqué dans la mienne de coupure. Mais le territoire n'est pas la carte» (Beauvoir [1963] 2018, I : 1218). Le territoire de la vie vécue n'est pas la carte du récit de vie : disposer le texte en chapitres, comme autant d'unités distinctes, à l'échelle d'une vie ou d'une période étendue, renvoie ainsi à des choix déterminants qui engagent la ressaisie du passé et la quête d'un sens.

De fait, les Mémoires de Beauvoir témoignent avec une grande acuité de l'importance $\mathrm{du}$ chapitre pour résoudre une tension décisive entre, d'une part, un récit historique essentiellement linéaire, expliquant comment les événements se sont succédé, comment les rencontres, anecdotes, expériences qui constituent le tissu d'une vie ont eu lieu les unes après les autres, et, d'autre part, une entreprise d' "institution de soi » analytique et même souvent dialectique, qui se donne sous le signe du projet, de la liberté, et qui instaure un régime de causalité singulier. Adossé à la chronique du temps passé (une jeunesse d'avant-guerre, puis la maturité des années 1940 aux années 1970), c'est ce régime de causalité qui permet de donner forme et sens à l'expérience collective de toute une génération aux prises avec l'Histoire, et de dessiner avec fermeté un itinéraire intellectuel, ou plutôt l'itinéraire d'un couple d'intellectuels dans le monde.

4 Des Mémoires d'une jeune fille rangée à la Cérémonie des adieux, Beauvoir s'essaie à diverses formes de l'écriture de soi : l'autobiographie proprement dite (Mémoires d'une jeune fille rangée, 1958), les Mémoires (La Force de l'âge, 1960 ; La Force des choses, 1963), l'autoportrait (Tout compte fait, 1972), le carnet de deuil (Une mort très douce, 1964 et La Cérémonie des adieux, 1981). Ainsi réuni - comme le manifeste pour la première fois de façon aussi nette l'édition des Mémoires dirigée par Jean-Louis Jeannelle et Éliane Lecarme-Tabone en 2018 -, ce corpus, dont la rédaction a couru sur près de vingt-cinq années, et qui récapitule plus de soixante-dix ans d'une existence intimement mêlée au $\mathrm{xx}^{\mathrm{e}}$ siècle, présente un effet sériel très net, du récit d'enfance jusqu'à celui de la mort de Sartre, en passant par les grandes expériences décisives : l'affirmation d'une vocation d'écrivain et d'intellectuelle, la conversion à l'Histoire, les affres de l'engagement politique, la mort de la mère, les désillusions idéologiques et la fin du couple. Tout cela est raconté en suivant le déroulement des faits, et soigneusement suturé grâce à un système d'échos et de renvois aux ouvrages précédents, avec quelques recouvrements partiels. L'ensemble constitue, en somme, un immense récit solidaire, la plupart du temps linéaire et chronologique, cherchant à restituer la totalité d'une expérience vécue, de la naissance jusqu'à la mort de Sartre. 
Ce corpus, pourtant réputé lisse et d'une ambition esthétique limitée, présente un intérêt particulier à la fois dans la perspective d'une histoire littéraire du chapitre, dans le cas des écritures de soi, et dans la perspective d'une analyse rhétorique de la capitulation, et ce, à quatre titres au moins. Comme dispositif de segmentation nécessaire du récit de vie, le chapitre apparaît d'abord comme l'une des modalités les plus visibles de la logique de récapitulation propre au vaste massif mémorial de Beauvoir : une unité à la fois chronologique et sémantique, marquant une série d'étapes ou de jalons dans le récit de vie. Mais le corpus présente une diversité remarquable d'usages du chapitre: Mémoires d'une jeune fille rangée est divisé en quatre parties, ellesmêmes composées de sections séparées par des blancs, sans chapitres nettement identifiés, donc ; La Force de l'âge et La Force des choses sont organisées en diptyque, deux parties précédées d'un prologue, La Force de l'âge comporte huit chapitres, La Force des choses onze chapitres, avec en sus un intermède et un épilogue, ces chapitres étant euxmêmes divisés en sections séparées par des blancs ou des astérisques; Tout compte fait compte huit chapitres, eux-mêmes subdivisés de façon plus lâche, mais qui rompent avec une organisation essentiellement chronologique; Une mort très douce est un récit continu, ne comportant que des sections séparées par des blancs, et La Cérémonie des adieux exhibe le déroulement chronologique par la succession des années, qui donnent leur titre à chaque section, sans saut de page, de 1970 à 1980. Le rapport au temps et les modalités mêmes du récit évoluent, tout au long de l'écriture; on verra ainsi que le chapitre en est l'un des symptômes et l'une des formes, comme ponctuation rythmique du récit, comme organisation des "méta-intrigues ${ }^{1}$ » qui donnent leur unité à chaque volume, et comme creuset d'une synthèse toujours inépuisable.

6 Une deuxième raison motivant l'intérêt porté au chapitre est qu'il est l'objet de réflexions explicites chez Beauvoir. Elle évoque à plusieurs reprises, dans des lieux stratégiques (prologue, intermède), la question de l'organisation séquentielle de son œuvre, et s'interroge sur la forme et l'enjeu des chapitres dans le récit de vie. On peut citer, entre autres, outre les formules frappantes de La Force de l'âge déjà évoquées, le prologue de La Force des choses:

Comme le précédent, ce livre demande au lecteur sa collaboration : je présente, en ordre, chaque moment de mon évolution et il faut avoir la patience de ne pas arrêter les comptes avant la fin. (Beauvoir [1963] 2018, I : 939)

auquel répond la fin de la première partie :

Le fond, tragique ou serein sur lequel mes expériences s'enlèvent leur donne leur vrai sens et en constitue l'unité ; j'ai évité de les lier par des transitions qui seraient univoques, donc artificielles. Alors puisque la totalisation me paraît si nécessaire, pourquoi me suis-je asservie à l'ordre chronologique au lieu de choisir une autre construction? J'y ai réfléchi, j'ai hésité. Mais ce qui compte avant tout dans ma vie, c'est que le temps coule; je vieillis, le monde change, mon rapport avec lui varie ; montrer les transformations, les mûrissements, les irréversibles dégradations des autres et de moi-même, rien ne m'importe davantage. Cela m'oblige à suivre docilement le fil des années. (Beauvoir [1963] 2018, I : 1218)

Il faut également ajouter l'intégralité du prologue de Tout compte fait, qui analyse et justifie l'abandon d'une organisation chronologique et le choix de chapitres thématiques (Beauvoir [1972] 2018, II : 487).

7 Le chapitre apparaît également comme une unité décisive dès la genèse des Mémoires, le cadre où s'éprouve le choix d'une structure signifiante - troisième raison de s'y attarder. Alors que, pour les Mémoires d'une jeune fille rangée ou La Force des choses, le chapitrage semble être intervenu assez tardivement dans la rédaction, répondant ainsi 
à un besoin de scander la coulée d'une écriture, de marquer les tournants d'une existence, dans La Force de l'âge et Tout compte fait, le chapitre est dès le début une unité d'écriture, matérialisé par la numérotation des pages et la distribution de liasses en rubriques nettement distinguées. Il y aurait donc des stratégies différentes, en termes de composition, qui se traduiraient potentiellement par un usage différencié du chapitrage. Dans un entretien datant de 1965 avec Francis Jeanson, Beauvoir reconnaît qu'elle procède par " totalisations partielles ${ }^{2}$ » : le chapitre est l'un des niveaux, dans le texte, où l'on peut observer à la fois la consignation de l'expérience effective, et sa réappropriation par le récit et l'analyse. C'est d'autant plus net quand on confronte le texte des Mémoires avec les Cahiers de jeunesse, pour les Mémoires d'une jeune fille rangée, ou avec les journaux, très partiellement cités et montés par Beauvoir dans $L a$ Force de l'âge et La Force des choses. Comme le souligne Annabelle Martin Golay (2013 : 155), « le matériau mémoriel a déjà fait l'objet d'une première élaboration à travers son journal, avec et contre lequel se construit le texte des Mémoires. Avec: car le texte mémorial se fonde sur du "déjà dit", sur une première transformation du réel. Contre: car il en défait la continuité propre pour lui substituer un ordre différent, qui agence et totalise ». Pour Tout compte fait, singulièrement, la redistribution en chapitres thématiques du matériau issu des journaux et des correspondances est l'un des gestes d'écriture les plus décisifs; certes, Beauvoir corrige son style, modifie localement l'ordre de certains paragraphes, mais l'essentiel de la campagne d'écriture consiste dans le développement de chapitres déjà constitués, ou dans le déplacement de chapitres. Ils sont l'unité essentielle de la composition ${ }^{3}$.

Le chapitre apparaît surtout, chez Beauvoir, comme le lieu où s'articulent une exigence chronologique - dessiner le cours d'une vie, des orientations d'un projet individuel à ses inscriptions dans l'histoire collective - et une réorganisation sémantique et dramatique des expériences passées. Certes, cette articulation n'est nullement spécifique à l'œuvre de Beauvoir. L'organisation séquentielle propre aux Mémoires, et plus largement aux récits factuels, répond en effet à une nécessité consubstantielle, comme l'a souligné Jean-Louis Jeannelle : «Ils visent à une forme d'exhaustivité et de continuité - en témoigne notamment l'impératif chronologique au nom duquel le déroulement suivi des faits vaut comme principale loi de causalité-, tout en se dérobant sans cesse à cette logique énumérative et accumulative, afin de ne pas se réduire à un simple catalogue de données indistinctes " (Jeannelle 2008: 355). Le chapitre est ainsi la forme la plus visible de la reconfiguration de l'effectif, à travers la composition d'un récit orienté. Il permet ainsi, pour reprendre à nouveau une formule de Jean-Louis Jeannelle, une "négociation ponctuelle» pour résoudre le «dilemme entre indexation de la réalité extradiscursive et unité de la représentation narrative » (Jeannelle 2008 : 357), restaurant, à un niveau intermédiaire, une forme d'intelligibilité du divers, et une ponctuation du flux continu de la temporalité historique. Or, la comparaison avec Les Mots de Sartre, dont les Mémoires d'une jeune fille rangée seraient le pendant féminin insuffisant selon quelques éminents critiques, en particulier, ou avec l'œuvre de Leiris, que Beauvoir cite beaucoup dans Tout compte fait, a pu conduire à négliger la composition des Mémoires de Beauvoir, trop rapidement réduite à une vaste explication chronologique. C'est particulièrement frappant chez Philippe Lejeune, dans Le Pacte autobiographique ou dans Les Brouillons de soi, qui considère Beauvoir comme une autobiographe naïve, incapable de masquer, sous la mince surface d'une structure dialectique forcée, la primauté d'une ligne de force avant tout chronologique et linéaire, chacun des chapitres du récit de sa vie ne faisant en somme que d'additionner 
infatigablement aux précédents et, semble suggérer Philippe Lejeune, assez vainement (Lejeune $1996: 200$ et suiv., 236-237 ; Lejeune $1998: 73-74,77$ et passim).

9 S'intéresser aux chapitres et aux modulations de leurs usages, dans l'ensemble du corpus, serait donc une manière de ressaisir l'ambition propre de Beauvoir touchant à la consignation de sa vie et à la représentation du temps passé, au-delà de la raillerie convenue sur son interminable enregistrement des faits et gestes du couple; c'est là le quatrième et déterminant enjeu d'un tel objet. Comme manière de saisir le temps et de restituer un certain rapport à la vie comme flux, le chapitre ne se résume pas à la manie de l'inventaire et de la liste d'anecdotes ou de faits historiques qui serait l'apanage de la compagne de Sartre. Problématisant «la totalisation possible et impossible » (Colin, Conrad et Leblond 2017 : 26), il se révèle un niveau pertinent pour mesurer les enjeux de la composition d'une œuvre réputée à peine composée. Réciproquement, les modulations de l'usage de la « capitulation ${ }^{4}$ » engagent un rapport singulier à la récapitulation, ouvrant un éventail de possibles au cœur de cette œuvre en apparence si verrouillée.

\section{Tournant, époque, tranches : ce que récapituler sa vie veut dire}

10 Dans son "Ouverture" au volume collectif sur les Pratiques et poétiques du chapitre, Philippe Hamon identifie «trois procédés de mise en ordre quasi-présémantique du monde : le rythme, la mise en récit et la mise en liste » (Colin, Conrad et Leblond 2017 : 13), qui finissent par apparaître comme autant de fonctionnalités possibles du chapitre ou de tout dispositif équivalent: scander le flux continu de l'existence, identifier des enchaînements logiques sous la trame chronologique des faits, inventer des rapports paradigmatiques entre des événements, des choses, pour ordonner le divers. Ces trois modalités de mise en ordre correspondent dans le récit beauvoirien à trois manières de désigner et de penser la segmentation de la vie même: l'époque, définie rythmiquement par des chapitres ou par une partie, le tournant, qui dramatise le passage d'une époque à une autre, et permet la structuration du récit, et enfin, la version la plus faible, la tranche, qui suggère finalement non seulement la contingence de toute forme de disposition, mais plutôt son caractère arbitraire - découpage inutile, au présent, d'un passé qui échappe, puisque c'est le flux, l'élan, l'expérience de la durée et le bonheur du projet qui fondent le goût de la vie pour Beauvoir ${ }^{5}$.

Dans Les Brouillons de soi, Philippe Lejeune avait évoqué l'idée d'une "grammaire des césures ", encore à construire, fatalement historique et comparative, pour penser le problème classique de la périodisation, même si, comme il le rappelle plus loin dans le même ouvrage : «Le montage est le problème essentiel du genre "souvenirs d'enfance", puisque la mémoire livre les souvenirs en désordre, et qu'en lui-même l'ordre chronologique ne saurait donner sens au récit » (Lejeune 1998: 107 et 263). Le niveau du chapitre semble donc particulièrement pertinent pour penser à la fois le tournant et l'époque - sous l'aspect faussement arbitraire de la tranche. 


\section{Les Mémoires d'une jeune fille rangée : naturaliser le romanesque - ou romancer le réel ?}

Dans les Mémoires d'une jeune fille rangée, on a donc quatre parties nettement identifiées, dont les articulations renvoient à des moments déterminants, presque toujours liés à l'institution scolaire, comme l'a montré Éliane Lecarme-Tabone ${ }^{6}$. Elles comportent de nombreuses séquences séparées par des blancs, où l'ordre chronologique se combine avec un ordre thématique (par exemple les lectures d'enfance, l'amitié avec Zaza, les rêves d'amour dans la deuxième partie). Le texte est à la fois fortement segmenté, en deçà du chapitre, avec ces séquences enchaînées, et nettement canalisé, au-delà du chapitre, avec ces quatre parties dépourvues de titre, qui fonctionnent comme une vaste structure dialectique ${ }^{7}$. On pourrait même parler d'un verrouillage rhétorique, à ce niveau. Le récit d'enfance et de jeunesse y répète quatre fois le même schéma, articulant conquête de l'autonomie et persistance d'une forme d'aliénation : bonheurs et impuissances enfantines pour la première partie; initiations et soumissions (lectures, amitié, amours), pour la deuxième partie ; progrès intellectuel et désillusions (la vie étudiante), pour la troisième partie; promesses d'avenir et avenir brisé (Sartre et Zaza), pour la quatrième partie. Il n'y a donc pas de chapitres à proprement parler dans ce récit inaugural, mais à la fois un morcellement rendant compte de la profusion des souvenirs d'enfance et donnant au récit l'épaisseur d'une chronique, et une armature dialectique donnant à lire une libération consciente et douloureuse. Une émancipation imparfaite au féminin, restituée à travers un canevas largement romanesque, dans ses motifs et ses lieux communs, mais reprise, au lieu d'une disposition chapitrale trop romanesque, dans cette structure dialectique très nette, qui " opère une dramatisation des tournants et une simplification des lignes " (Beauvoir [1958] 2018, I : 1232).

\section{La Force de l'âge : de la naturalisation des époques à la dramatisation du tournant}

Dans La Force de l'âge, des chapitres nettement identifiés, dûment numérotés, constituent un niveau supplémentaire dans le dispositif, en sus de la distribution macrostructurale en parties et de la segmentation par des blancs. Il y a sans doute d'abord une motivation d'ordre quantitatif, le texte étant environ $50 \%$ plus long que le précédent. Mais surtout, ce que Beauvoir présente elle-même dans le prologue comme une "suite » nécessaire relève d'une logique différente. Elle est passée de «l'histoire d'une vocation » à son incarnation. La perspective est ainsi moins généalogique que téléologique. Dans la genèse de La Force de l'âge, on peut observer la transformation progressive d'un programme narratif (écrire la suite de sa vie) en intention pleinement analytique (exposer clairement les postulations contradictoires de deux périodes de sa vie, séparées par le grand tournant de la guerre, qui marque la fin des aspirations idéalistes de la jeune agrégée de philosophie, et la conversion à l'Histoire et à l'écriture authentique). Cette perspective analytique est nettement portée, là encore, par une macrostructure : la division en deux parties, la première allant jusqu'à août 1939, la seconde reprenant en septembre 1939. On retrouve, à l'échelle des sections, l'articulation entre des jalons chronologiques et des micro-synthèses thématiques, sur ses rencontres, ses lectures, ses projets d'écriture, les événements historiques, etc. 
14 À quoi servent finalement les chapitres, par rapport à la structure des Mémoires d'une jeune fille rangée ? On l'a déjà souvent remarqué, l'unité des chapitres dans La Force de l'âge tient à ce qu'ils suivent le rythme des années scolaires, de la rentrée aux vacances d'été, sans pour autant s'y couler parfaitement. Ainsi, les chapitres un, trois et quatre recouvrent deux années scolaires; le chapitre deux en retrace une seule (l'année déterminante pour Beauvoir de sa titularisation comme professeure à Marseille), le chapitre cinq en embrasse trois (1936 à 1939 : Beauvoir garde le même poste - c'est la marche à la guerre). La partie deux obéit à une structure ternaire inégale : une année, pour le chapitre six (la défaite), puis trois, pour le chapitre sept (l'Occupation), puis deux pour le dernier chapitre (les promesses de la Libération). Chaque chapitre se clôt ainsi sur l'évocation des vacances et donc des voyages de Beauvoir, avec ou sans Sartre, "véritable ponctuation chronologique (conformément au calendrier scolaire) et narrative (puisque ces échappées offrent une phase intermédiaire entre la sphère personnelle et le contexte historique), ils sont la condition d'une épiphanie perpétuellement renouvelée ${ }^{8} »$.

15 La macrostructure soulignant la conversion brutale de Beauvoir à l'Histoire est ainsi tempérée par ces retours cycliques manifestant son goût de vivre, son rapport au monde sous le signe du bonheur. On pourrait aussi voir dans cet enchaînement des chapitres comme autant d'années scolaires, la restitution ironique d'une expérience routinière authentiquement vécue: on aurait ainsi la négation de la violence de l'événement historique, dans la première partie, l'évocation d'un refuge précaire dans la seconde, le dispositif soulignant cruellement un rapport au temps immature. Loin d'être arbitraire ou essentiellement téléologique, le chapitre apparaît ainsi comme une scansion intermédiaire entre la pure accumulation thématico-chronologique et l'organisation rhétorique des parties : il permet de saisir à la fois des moments dans le flux d'une vie, et d'en exposer ironiquement les illusions.

\section{La Force des choses : naturaliser les emportements de l'Histoire?}

16 Avec La Force des choses, le temps de l'écriture se rapproche du temps raconté, jusqu'à s'y confondre pour finir. Beauvoir mobilise les journaux qu'elle a tenus de la Libération à la fin des années 1950, en particulier pour tout ce qui touche à la guerre d'Algérie, et le chapitrage semble être intervenu plus tardivement dans la genèse. Un prologue précède deux parties séparées par un intermède, suivies par un épilogue, les deux parties comportant respectivement cinq et six chapitres, qui sont les plus longs de tout le corpus. L'inflation est cependant à relativiser, puisque, là encore, le texte est nettement plus long que le précédent, prolongeant un très net mouvement de prolifération de l'écriture mémorielle. Les scansions, fortement marquées, sont associées à des tournants à la fois intimes et collectifs : la grande coupure de 1952, entre les deux parties du récit, correspond à la fois à la conversion de Sartre au communisme, à la rupture avec Algren, son amant américain, et à la rencontre avec Lanzmann, ainsi qu'à la prise de conscience d'un vieillissement inéluctable. L'enchaînement des chapitres ne répond plus au calendrier scolaire, mais de façon plus irrégulière, il rend compte d'une série de désillusions : tandis que le premier chapitre s'ouvre sur les promesses de la Libération, les clausules et les seuils des chapitres suivants thématisent fortement le départ, l'échec, le changement, les espérances démenties par l'Histoire. 
17 Alors que les deux grandes parties semblent fonctionner en miroir, de la honte de la trahison des intellectuels français après la Libération à la honte de la guerre d'Algérie, et enregistrent la radicalisation des engagements de Beauvoir et de Sartre, le dispositif des chapitres déjoue toute tentative de restaurer un rythme régulier permettant d'habiter l'Histoire. L'engagement éthique et politique sans cesse réaffirmé se heurte aux démentis amers; la confusion relative de la chronologie des émotions, des rencontres amoureuses, des projets littéraires se croise avec la chronologie d'une Histoire dont la mémorialiste enregistre chaque soubresaut. Aucune synthèse dialectique ne sera possible, malgré l'armature rhétorique de l'ensemble.

\section{Neutraliser la (ré)-capitulation : nouvelles modalités pour finir (Tout compte fait et les carnets de deuil)}

Tout compte fait propose une troisième modalité de chapitrage, nettement expliquée par Beauvoir elle-même dans un bref prologue; elle y affirme renoncer à l'ordre chronologique, pour proposer une série de chapitres thématiques - en réalité, seul le premier chapitre est véritablement synchronique, les autres chapitres sont portés par un fil chronologique, et ressortissent plutôt à la catégorie de la rubrique : écriture et lecture, voyages d'agrément, voyages politiques, activités militantes diverses, qui fonctionnent comme autant de codicilles, pour la période considérée (1962-1972), à ce qui a été raconté dans les volumes précédents.

Dans Une mort très douce et La Cérémonie des adieux, la disparition des chapitres tient sans doute d'abord à la brièveté des textes, mais surtout à l'exposition de la violence d'un temps inéluctable, celui de l'agonie; à chaque section correspond une étape qui rapproche la mère, puis Sartre de la mort. La sécheresse de l'exposé se dérobe à toute forme de récupération narrative, au sens d'un récit organisé, rationalisé. Seuls valent ici la pure succession des faits et l'enregistrement des émotions, à leur date.

De fait, l'écriture des Mémoires ne cesse de négocier cette tension vive entre l'angoisse d'un temps qui échappe, la violence de l'insaisissable, de l'irrémédiable, et l'aspiration sans cesse renouvelée au récit juste-celui qui trouve le bon rythme de la récapitulation. La Force des choses révèle cette tension majeure au détour d'un récit de rêve, au milieu du chapitre XI de la seconde partie :

Cette nuit, un rêve d'une extrême violence. Je suis avec Sartre dans ce studio; le phono repose sous son voile. Soudain musique, sans que j'aie bougé. Il y a un disque sur le plateau, il tourne. Je manœuvre le bouton d'arrêt: impossible de l'arrêter, il tourne de plus en plus vite, l'aiguille ne peut pas suivre, le bras prend d'extraordinaires positions, l'intérieur du phono ronfle comme une chaudière, on voit des espèces de flammes, et le luisant du disque noir, affolé ; d'abord l'idée que le phono va se détraquer, une angoisse limitée, puis qui devient immense : TOUT va exploser; une rébellion magique, incompréhensible, c'est un dérèglement de tout. [...] la force indocile et mystérieuse c'était celle du temps, des choses, elle dévastait mon corps (ce misérable rogaton de bras desséché), elle mutilait, elle menaçait de radical anéantissement mon passé, ma vie, tout ce que j'étais. (Beauvoir [1963] 2018, II : 307) 


\section{Récapituler : ressaisir le temps passé ou décompter les chapitres d'une vie}

21 Pour Francis Jeanson, "nous nous essoufflons à suivre, d'épisode en épisode, le fil de son existence : et s'il est vrai qu'il y a beaucoup de "vie" dans cette prodigieuse énumération, du moins sommes-nous contraints d'y ressentir le présent sous la forme d'une certaine absence - comme dans toute histoire dont nous ne pouvons pas ignorer qu'elle s'est déjà inscrite, quelque part, dans le monde réel » (Jeanson 1966: 235). Faut-il ainsi comprendre cette entreprise de récapitulation comme une «prodigieuse énumération ", comme une pure succession d'épisodes? Il semble que l'analyse du fonctionnement des chapitres comme niveau intermédiaire entre les épisodes (des diverses sections), les inventaires, les séquences nettement identifiées (récits de voyage, portraits, anecdotes, analyses de son travail d'écrivain...) invite à nuancer ce constat. Au-delà d'une "vision synoptique rétrospective " associant méta-intrigue (s'émanciper de sa classe petite-bourgeoise, se convertir à l'Histoire et à l'écriture, affirmer son engagement intellectuel contre toutes les formes de compromissions historiques) et inventaire de la vie vécue, individuelle et collective, c'est-à-dire fondée à la fois sur la structure dialectique d'ensemble et sur les détails profus dont ces quelque deux mille pages font l'inventaire, la scansion du chapitre tente de ressaisir un temps qui n'est pas encore constitué.

Le chapitre déjouerait ainsi d'autres logiques, qui seraient celles de l'explication, du grand système dialectique, ou d'une téléologie sèche, mais sans pour autant restaurer le sentiment illusoire d'une vie ouverte à toutes les potentialités. Beauvoir a sans nul doute médité la réflexion de Merleau-Ponty, dont elle était encore proche en 1945, quand paraît sa Phénoménologie de la perception: "Le temps constitué, la série des relations possibles selon l'avant et l'après, ce n'est pas le temps même, c'en est l'enregistrement final, c'est le résultat de son passage que la pensée objective présuppose toujours et ne réussit pas à saisir. [...] Il doit y avoir un autre temps, le vrai, où j'apprenne ce que c'est que le passage ou le transit lui-même » (Merleau-Ponty 1945 : 474-475). Il ne faut pas confondre la carte et le territoire, le balisage narratif et la dynamique propre au présent.

Ce que le chapitre prend en charge, dans une économie complexe où se croisent divers dispositifs de segmentation du texte, c'est un mouvement de totalisation partielle, toujours inachevée, qui exhibe l'hétérogénéité résistante de l'expérience vécue. Il ne s'agit pas pour Beauvoir de mimer une écriture à suspense, pour rouvrir vainement les potentialités d'une temporalité déjà advenue, mais bien plutôt de suggérer la forme heurtée du temps vécu, son ambiguïté essentielle9. Pour Raphaël Baroni, «[n]ous ressentons les ressacs du temps, ses ouvertures, ses durées et ses fermetures, ses débuts, ses milieux et ses fins, parce que, pour un être sensible, les choses qui arrivent s'arrachent au temps planifié du calendrier, elles ne sont pas une perpétuelle répétition du même et ne se suivent pas nécessairement dans un fondu-enchaîné » (Baroni 2009 : 119).

Comment comprendre, dans cette perspective, les modulations du dispositif chapitral, tout au long de la rédaction des Mémoires ? Peut-être peut-on pour finir proposer une hypothèse, ou plutôt une reconfiguration rêveuse de l'ensemble des Mémoires, lus comme succession de dispositifs pour ressaisir le temps vécu, au passé, qui témoignent d'une lente dé-dialectisation du récit - et par là même d'une reformulation 
mélancolique d'un rapport au politique et à l'écriture de soi dans l'Histoire. Dans son dernier ouvrage, Les Bords de la fiction, paru en 2017, Jacques Rancière propose un radical déplacement de la définition politique de la fiction, dans la continuité de ses travaux antérieurs, mais en insistant sur la manière dont elle reconfigure le temps, à travers ses enchaînements, son organisation séquentielle. Rancière explique ainsi que la fiction, telle que l'a conceptualisée Aristote, rationalise la temporalité, au prix d'une hiérarchisation de facto politique des formes de vie, "qui distingue les hommes "actifs" des hommes "passifs" par leur manière d'habiter le temps, par le cadre sensible de leur activité et de leur inactivité » (Rancière 2017 : 149). Aristote associait ainsi un certain type de forme de vie à l'organisation narrative de la fiction : "La rationalité poétique des enchaînements nécessaires ou vraisemblables s'applique à des hommes que l'on dit actifs parce qu'ils vivent dans le temps des fins : celles que l'action se propose mais aussi cette fin en soi que constitue la forme privilégiée d'inaction nommée loisir » (Ibid.).

N'est-ce pas là une définition redoutable non seulement du héros, dans ses avatars traditionnels, mais aussi de l'intellectuel, tel qu'il se caractérise au $x^{e}$ siècle, et tel que Beauvoir et Sartre peuvent l'incarner exemplairement, à la fin de La Force de l'âge et dans La Force des choses? Pour Rancière, la fiction véritablement moderne - il donne les exemples de Woolf ou de Sebald, bien éloignés de Beauvoir évidemment - doit sortir des modèles téléologiques décrits par Aristote, s'exonérer d'un traitement rationnel du temps, de ce que Rancière nomme sa "fonction mesquine d'explication " (à travers les catégories du «nécessaire» et du "vraisemblable»), pour trouver une autre façon "d'identifier des événements et de les lier les uns aux autres » :

La révolution démocratique de la fiction n'est pas le grand surgissement des masses sur la scène de l'Histoire. Elle n'en est pas moins fidèle à la définition moderne de la révolution : celle-ci est le processus par lequel ceux qui n'étaient rien deviennent tout. Mais devenir tout, dans l'ordre fictionnel, ce n'est pas devenir le personnage principal de l'histoire. C'est devenir le tissu même au sein duquel - par les mailles duquel - des événements tiennent les uns aux autres. (Rancière 2017 : 152)

Dans cette perspective, on pourrait lire, à travers les jeux de chapitrage des Mémoires, la conquête perturbée d'un dispositif rationnel, puis son délitement. Comme lieux et comme rythmes de la perturbation, les chapitres viennent scander l'expérience vécue pour défaire la dimension téléologique ou dialectique des récits. Quand il ne reste plus que les chapitres, qui subsument une linéarité devenue seconde (Tout compte fait), et puis quand les chapitres s'effacent eux-mêmes pour ne laisser place qu'à l'enregistrement du temps qui précipite l'être aimé vers la mort (Une mort très douce et La Cérémonie des adieux), c'est la figure de l'intellectuelle qui se brouille, pour se dépouiller de ce qui l'avait constituée comme telle - la conversion à l'Histoire, les engagements politiques. Une autre écriture de l'Histoire se donne finalement à lire, mélancolique, inscrivant au cœur des Mémoires une interrogation sensible sur l'objet même du mémorable : l'enchevêtrement irréductiblement singulier des émotions, des expériences historiques et personnelles, des projets et des désillusions d'un individu emporté dans le temps.

Cela se fait-il au prix de la lisibilité ? Peut-être, dans le cas de Tout compte fait, qui n'est sans doute plus lisible que par élans ou par morceaux choisis, ou bien au contraire en surplomb, dans une perspective macrostructurale; mais certainement pas pour Une mort très douce ou La Cérémonie des adieux. En s'éloignant de l'efficacité rationnelle de la dialectique, en esquivant à la fois les formes séduisantes de la fragmentation et les recompositions dramatiques, Beauvoir n'a pas choisi naïvement l'esthétique du 
«magnétophone». Si les Mémoires sont bien la «reconfiguration narrative du mémorable d'une vie, transmise dans le souci de servir à la connaissance des mobiles qui guident les hommes engagés dans le cours de l'Histoire" (Jeannelle 2008: 392), le mémorable s'est progressivement trouvé un rythme, pour dire la forme d'une vie vécue, vivre dans le temps des fins, y éprouver le glissement d'un absolu intime (Mémoires d'une jeune fille rangée) à une exigence éthique (La Force de l'âge) jusque dans ses contradictions historiques (La Force des choses) et enfin dans l'aporie (Tout compte fait) - et le ressaisir enfin dans une écriture fortement scandée, chapitrant les angoisses existentielles et tenant tête à l'oubli, comme au mépris.

\section{BIBLIOGRAPHIE}

Baroni, Raphaël, 2009, L'œuvre du temps, Paris, Seuil, coll. « Poétique ».

Beauvoir, Simone de, 2018, Mémoires, I et II, éd. Jean-Louis Jeannelle et Éliane Lecarme-Tabone, Paris, Gallimard, coll. « Bibliothèque de la Pléiade ».

Brainbridgge, Susan, 2005, Writing against Death. The Autobiographies of Simone de Beauvoir, Amsterdam, Rodopi, coll. « Faux titre ».

Colin, Claire, Conrad, Thomas et Leblond, Aude (dir.), 2017, « Introduction générale », Pratiques et poétiques du chapitre, du XIX $x^{e}$ au XXI $I^{e}$ siècle, Rennes, PUR.

Dionne, Ugo, 2008, La Voie aux chapitres, Poétique de la disposition romanesque, Paris, Seuil, coll. «Poétique ».

Jeannelle, Jean-Louis, 2008, Écrire ses Mémoires au XXe siècle, Déclin et renouveau, Paris, Gallimard, coll. «Bibliothèque des idées ».

Jeannelle, Jean-Louis, 2013, "Écrire ses Mémoires : récit de formation et "devoirs virils" », dans J.-L. Jeannelle et É. Lecarme-Tabone (dir.), Simone de Beauvoir, Paris, Éditions de l'Herne, coll. « Cahiers de l'Herne », p. 234-240.

Jeanson, Francis, 1966, Simone de Beauvoir ou l'entreprise de vivre, Paris, Seuil.

Kruks, Sonia, 2012, Simone de Beauvoir and the Politics of Ambiguity, Oxford, New York, Oxford University Press, coll. « Studies in Feminist Philosophy ».

Lecarme-Tabone, Éliane, 2000, Éliane Lecarme-Tabone commente les Mémoires d'une jeune fille rangée, Paris, Gallimard, coll. « foliothèque ».

Lejeune, Philippe, 1996, Le Pacte autobiographique, nouv. éd. augm., Paris, Seuil, coll. « Points essais ».

Lejeune, Philippe, 1998, Les Brouillons de soi, Paris, Seuil, coll. « Poétique ».

Martin Golay, Annabelle, 2013, Beauvoir intime et politique, la fabrique des Mémoires, Lille, Presses universitaires du Septentrion.

Merleau-Ponty, Maurice, 1945, Phénoménologie de la perception, Paris, Gallimard.

Rancière, Jacques, 2017, Les Bords de la fiction, Paris, Seuil, coll. « La bibliothèque du XxI ${ }^{\mathrm{e}}$ siècle ». 


\section{NOTES}

1. Selon la formule de Jean-Louis Jeannelle (2013: 234-240).

2. Dans un entretien avec Francis Jeanson, reproduit dans Simone de Beauvoir ou l'entreprise de vivre (1966: 286).

3. Sur ces questions, on pourra se reporter à la notice de Tout compte fait, dans Mémoires, II, ainsi qu'à la notice de Jean-Louis Jeannelle, pour La Force des choses, dans Mémoires, I.

4. Pour reprendre le terme d'Ugo Dionne (2008), dont les analyses historiques et poétiques sont pionnières.

5. Susan Brainbridgge (2005) a montré combien étaient liés l'écriture comme geste et ce goût de la vie comme élan dynamique, tout contre la mort.

6. On pourra se reporter à Éliane Lecarme-Tabone commente les Mémoires d'une jeune fille rangée (2000) et aux indications fournies dans la notice procurée par la même auteure dans l'édition des Mémoires, I, qui ont largement inspiré la brève analyse proposée ici.

7. Éliane Lecarme-Tabone, « Notice » des Mémoires d'une jeune fille rangée, Mémoires, I, p. 1228.

8. Jean-Louis Jeannelle, « Notice » de La Force de l'âge, Mémoires, I, p. 1298.

9. Sur cette question de l'ambiguïté, décisive chez Beauvoir, on se reportera à Sonia Kruks (2012).

\section{RÉSUMÉS}

À partir des Mémoires de Simone de Beauvoir, on se propose d'analyser comment l'articulation des chapitres joue le rôle d'un dispositif essentiel, pour organiser la totalisation partielle des expériences vécues, et pour donner forme au récit, entre récapitulation et reconfiguration du sens d'une vie. La distribution en chapitres varie selon l'enjeu propre de chacun des volumes qui constituent les Mémoires: elle souligne la dimension romanesque de Mémoires d'une jeune fille rangée, accentue les enjeux dialectiques de La Force de l'âge (entre dramatisation du tournant et chronique d'une époque) et de La Force des choses (entre rhétorique et prolifération de l'écriture), et cristallise la vocation synthétique de Tout compte fait. Le dispositif capitulaire est l'une des structures qui rendent sensibles l'évolution d'un rapport au temps intime et politique, et à la forme d'une vie, transcrite sous le signe d'une liberté dynamique jusqu'à son accomplissement mélancolique.

Based on Simone de Beauvoir's Memoirs, this article studies the way chapters organize the "partial totalization" of experience, which functions both as a recapitulation and reconfiguration of the significance of life. The distribution in chapters varies according to the specific issue of each volume: it underlines the novelistic dimension of Memoirs of a Dutiful Daughter, emphasizes the dialectical issues of The Prime of Life-which dramatizes the turning points of her existence while chronicling an era-and Force of Circumstances-where the rhetoric structure balances the narrative proliferation-, and shows the challenges of synthesis in All said and done. As a structure, the chapter shows the evolution of an intimate and political relationship with time, 
and with the form of a life which is at first characterized by a dynamic freedom, until it reaches its melancholic conclusion.

INDEX

Mots-clés : Beauvoir (Simone de), Mémoires, écriture de soi, écriture de l'histoire, chapitre, forme de vie

Keywords : Beauvoir (Simone de), Memoirs, autobiography, history writing, chapter, forms of life

\section{AUTEUR}

HÉLÈNE BATY-DELALANDE

Université de Paris, CERILAC 